This item was submitted to Loughborough's Research Repository by the author.

Items in Figshare are protected by copyright, with all rights reserved, unless otherwise indicated.

\title{
Label-free chemical/biochemical sensing device based on an integrated microfluidic channel within a waveguide resonator
}

PLEASE CITE THE PUBLISHED VERSION

https://doi.org/10.1109/estc.2010.5642874

PUBLISHER

IEEE

VERSION

AM (Accepted Manuscript)

\section{PUBLISHER STATEMENT}

(c) 2010 IEEE. Personal use of this material is permitted. Permission from IEEE must be obtained for all other uses, in any current or future media, including reprinting/republishing this material for advertising or promotional purposes, creating new collective works, for resale or redistribution to servers or lists, or reuse of any copyrighted component of this work in other works.

\section{LICENCE}

\section{All Rights Reserved}

\section{REPOSITORY RECORD}

McKeever, E, SK Pavuluri, R Lopez-Villarroya, G Goussetis, DM Kavanagh, Mazher Mohammed, and MPY Desmulliez. 2010. "Label-free Chemical/biochemical Sensing Device Based on an Integrated Microfluidic Channel Within a Waveguide Resonator". Loughborough University. https://hdl.handle.net/2134/12627512.v1. 


\title{
Label-free Chemical/Biochemical Sensing Device based on an Integrated Microfluidic Channel within a Waveguide Resonator
}

\author{
E. McKeever*1, S. Pavuluri ${ }^{1}$, R. Lopez-Villarroya ${ }^{1}$, G. Goussetis ${ }^{2}$, D. M. Kavanagh ${ }^{1}$, M. I. Mohammed ${ }^{1}$, and M. P. Y. \\ Desmulliez $^{1}$ \\ ${ }^{1}$ Microsystems Engineering Centre (MISEC), School of Engineering and Physical Sciences, Heriot-Watt University, \\ Edinburgh, EH14 4AS, UK \\ ${ }^{2}$ ECIT, Queen's University Belfast, N. Island Science Park, Queen's Road, \\ Queen's Island, BT3 9DT, North Ireland \\ E-mail: em161@hw.ac.uk
}

\begin{abstract}
In this article we propose a novel label-free chemical/biochemical sensing device based on a waveguide resonator with an integrated microfluidic channel. This device is intended for the characterisation and detection of cells and various chemical substances from within a variety of micro-litre test samples. This paper outlines the design of the prototype device and describes the fabrication of the microfluidic network and microwave resonator. Initial testing of the prototype at Xband is examined with promising results. Finally, we propose a Low Temperature Co-fired Ceramic (LTCC) integration scheme for the miniaturization of the device for use in the $\mathrm{THz}$ regime.
\end{abstract}

\section{Introduction}

Microfluidics is the use of micro-scale devices to perform laboratory processes in micro-, nano-, and even pico-litre volumes. Such devices are manufactured using microfabrication technology to produce channels, pumps, and valves to manipulate, mix and analyze solutions $[1,2,3]$. Biosensors are a particular application that has flourished with the development in microfluidics. These systems use chemical, electrical or optical detection techniques for rapid sensitive analysis of samples. One commercially available biosensor is the glucose monitor [4], which takes micro-litre volumes of blood and determines the concentration of glucose in the test sample. Such biosensors generate a detectable signal, utilising reaction chemistries that require the attachment of a suitable fluorophore or enzymatic label. Consequently, this alteration of the sample from it native form makes it unsuitable for further analysis and may also alter the biological function of the sample itself.

The use of electromagnetic (EM) waves to characterize and study the dielectric properties of biological substances such as tissues and cell suspensions is well established through the work of Schwan [5]. In his work, he identified three distinct regions in which the electrical properties of tissues and cell suspension change with frequency. They are termed the $\alpha-, \beta-$, and $\gamma-$ dispersions pertaining from low frequencies $(<100 \mathrm{~Hz})$, intermediate RF frequencies (around $100 \mathrm{~Hz}$ to $10 \mathrm{MHz}$ ) and frequencies above $1 \mathrm{GHz}$.

The use of EM waves offers a number of advantages including a non-invasive, contactless label-free detection solution, with the ability to achieve fast measurements and precise characterization. With today's fabrication techniques and advances in microfluidics the development of lab-on-a-chip (LOC) devices has become available.

We propose in this article to integrate a microfluidic channel within a waveguide-based resonator with localized increased near-fields and sharp resonant features for label-free chemical/biochemical sensing.

\section{Prototype Sensor}

For initial testing and proof of concept we developed and tested a prototype sensor based on a waveguide, operating in the X-band, and with a precision machined entrance for insertion of the integrated microfluidic channel.

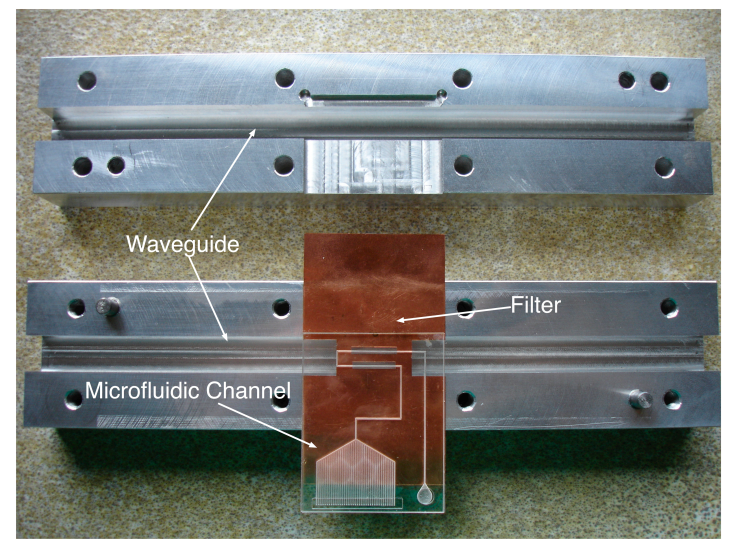

Figure $1 \mathrm{X}$-band waveguide halved along the E-Plane with insert for filter and microfluidic channel

The waveguide is machined from aluminium with waveguide WR-90 dimensions. The waveguide is mechanically halved along the E-plane for the insertion of the filter and microfluidic channel, as shown in Figure 1.

A finite element method (FEM) software package available from Ansoft [6] was used to design the filter as shown in figure 2. The filter has two resonators, which introduce two resonances as we can see in Figure 3. If the two resonators are identical then the two resonances will become degenerate and not distinguishable. By having slightly different lengths we can make the two resonances distinguishable. As the two coupled resonances cancel out at a certain frequency and it is here you have the transmission zero. The combined effect leads to a sharp feature that can be exploited in sensing. 


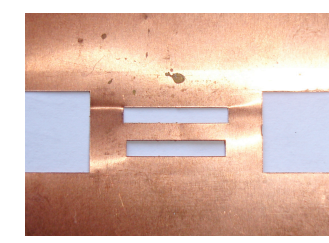

Figure 2 Micromachined copper filter

To optimise the sharp response and bandwidth we performed a study on the relative lengths of the resonators with the top resonator measuring $13 \mathrm{~mm}$ and the bottom resonator varying from $12 \mathrm{~mm}$ to $13 \mathrm{~mm}$ in $0.25 \mathrm{~mm}$ increments. The results of this study are shown below in Figure 3.

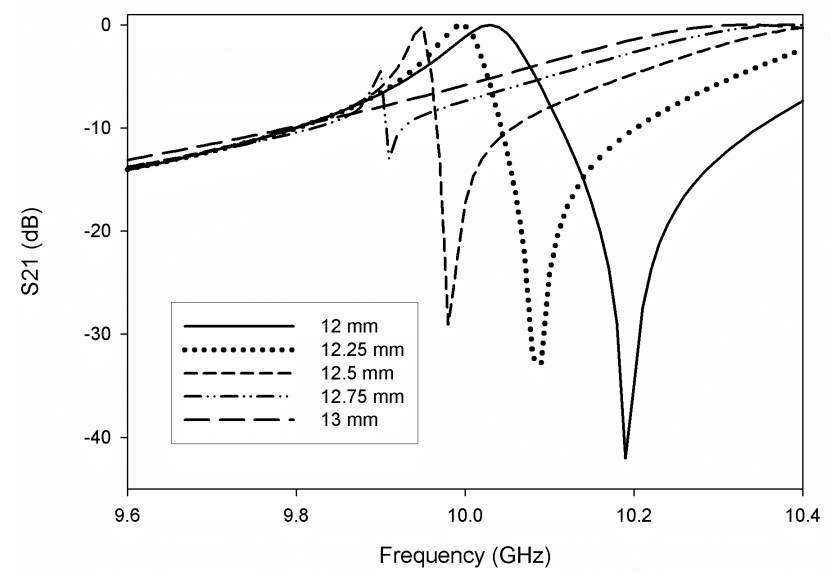

Figure 3 Bottom resonator length study in HFSS

An optimum length of $12.25 \mathrm{~mm}$ for the bottom resonator was chosen as a trade-off between the sharpness of the transmission response and the absorption losses. The resulting transmission pole is at approximately $9.99 \mathrm{GHz}$ with $-0.091 \mathrm{~dB}$ and the transmission bandwidth is $90 \mathrm{MHz}$ with a sharp roll-off. The narrow bandwidth allows for a higher sensitivity in sensing.

The microfluidic channels were fabricated from Polymethylmethacrylate (PMMA), which is sold under various trade names such as Plexiglas ${ }^{\circledR}$. Using an Epilog Legend Elite Series $\mathrm{CO}_{2}$ laser system [7] we engraved $350 \mu \mathrm{m}$ deep channels. The channel structure is shown in Figure 4; it consists of an inlet port, two parallel channels which align with the resonators and a microfluidic pump at the outlet. This microfluidic pump works on natural capillary forces that draw the fluid from the inlet to the vent (outlet) [8]. The chip was then bonded to another sheet of PMMA with access holes for the inlet and outlet.

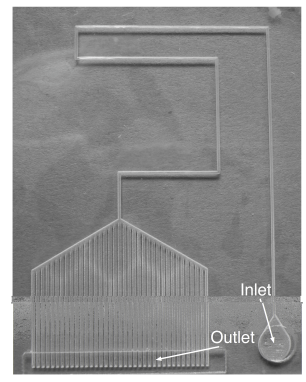

Figure 4 PMMA laser machined microfluidic channel

\section{Preliminary Results}

The waveguide was connected to the connectors of a HP 8510 Vector Network Analyser (VNA) as shown in Figure 5.

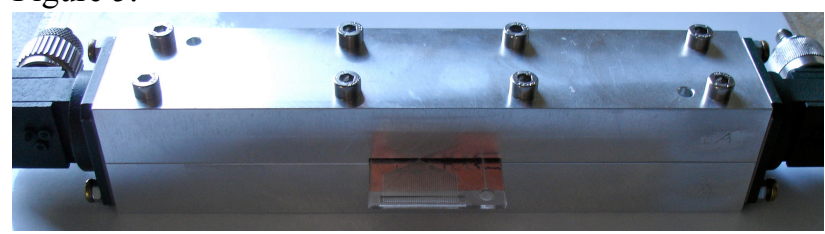

Figure 5 Waveguide showing connections to VNA and insert of the filter and microfluidic channel

The forward transmission coefficient, $\mathrm{S}_{21}$, was measured for an empty channel and a channel filled with Phosphate Buffer Saline (PBS). PBS is an isotonic salt solution used to dilute blood due to its use maintaining cellular osmotic integrity and a constant test sample $\mathrm{pH}$. The results are shown below in Figure 6.

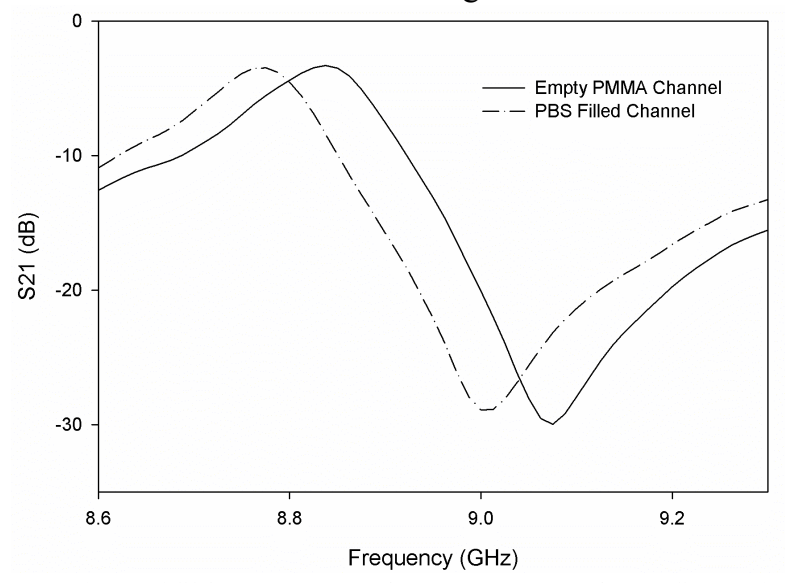

Figure 6 S21 parameter v. frequency results for $\mathrm{PBS}$ and air filled empty channel

As we can see there is significant difference between the empty channel and when it is filled with PBS. The resonant frequency of the empty channel is $9.8375 \mathrm{GHz}$ whereas when filled with PBS it becomes $9.775 \mathrm{GHz}$. The difference is $62.5 \mathrm{MHz}$, which is more than sufficient in distinguishing between the two media.

\section{LTCC Integration}

We also propose a packaging technique to allow the miniaturisation of the device for use in the $\mathrm{THz}$ regime. Low Temperature Co-fired Ceramic (LTCC) is a multilayer manufacturing platform that exhibits good electromagnetic immunity, which is therefore used primarily in microwave electronics. The process uses a material, when unfired, is called green tape, where individual sheets are processed in parallel then aligned and stacked prior to firing at a temperature below $1000^{\circ} \mathrm{C}$. This temperature allows the integration of highly conductive metals such as gold and silver that cannot be realised at higher temperatures. As the sheets are all fired together this reduces the overall manufacturing cost, saves time. Due to the dielectric constant of the material the dimensions of the resulting circuits can also be reduced. Another advantage of this technology is the parallelism in which every layer can be inspected before 
firing ensuring that one does not have to manufacture an entirely new device.

LTCC exhibits very good electrical and mechanical properties, high reliability and stability with the capability of manufacturing three-dimensional (3-D) structures. It has made its way into many applications, one such area of interest is Biological MicroElectroMechanical Systems (BioMEMS). This area uses the concepts of MEMS and applies them to biology to create examples such as microfluidics [9], PCR systems [10], biosensors [11], and bioreactors [12].

LTCC is also used to manufacture transmission lines and waveguides. Substrate Integrated Waveguides (SIW) are a form of transmission line in which vias are either embedded into a dielectric substrate, [13], or pillars are erected on top of the substrate, [14].

Here we propose a substrate integrated waveguide of the pillars in air design. The integration of our filter and microfluidic channel is shown in Figure 7.

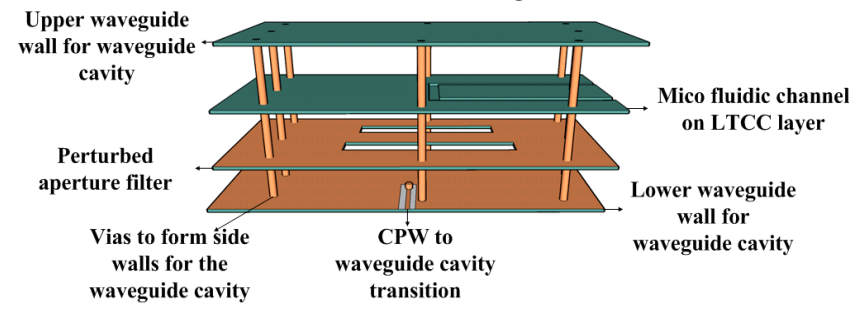

Figure 7 Proposed LTCC post wall waveguide design with integrated filter and microchannel

The sidewalls of the cavity are realised in closely spaced pillars with tape layers for the top and bottom. The microfluidic channel and filter can be realised in layers of ceramic material and embedded within the cavity. The waveguide is fed with a CoPlanar Waveguide (CPW) to waveguide cavity transition as shown in the diagram.

\section{Conclusions}

A prototype microwave waveguide cavity with integrated microfluidics sensor has been discussed. The preliminary results shown were promising and allow the detection of micro-litre volumes of liquid within the channel.

A packaging technique has also been proposed for application at higher frequencies, which could potentially enhance the sensing device making it capable of outperforming the low throughput of detecting and counting cells associated with optical techniques and could offer higher sensitivity compared to lower frequency and DC devices.

\section{References}

1. Cheng, X, "A Microfluidic device for practical labelfree CD4+ $\mathrm{T}$ Cell Counting of HIV-Infected Subjects", Lab on a Chip, Vol. 7 (2007), pp. 170-178.

2. Cheng, X, "Cell Detection and Counting Through Cell Lysate Impedance Spectroscopy in Microfluidic Devices", Lab on a Chip, Vol. 7 (2007), pp. 746-755.
3. Weigl, B H, Bardell, R L, and Cabrera, C R, "Lab-ona-chip for Drug Development", Adv. Drug Del. Rev., Vol. 55, Issue 3 (2003), pp. 349-377.

4. The Global Diabetes Community, Guide to Blood Glucose Meters, www.diabetes.co.uk

5. Schwan, H P, "Electrical Properties of Tissues and Cell Suspensions: Mechanisms and Models", Engineering in Medicine and Biology Society, Vol. 1 (1994), pp. A70-A71.

6. Ansoft high-performance EDA software, ANSYS Inc., www.ansoft.com

7. Epilog Legend Elite Series Laser, Epilog Laser, www.epiloglaser.com

8. Juncker, D, et al., "Autonomous Microfluidic Capillary System”, Anal. Chem., Vol. 74 (2002), pp. 6139-6144.

9. Birol, H, Maeder, T, and Ryser, P, "Low Temperature Co-fired Ceramic (LTCC) Technology: General Processing Aspects and Fabrication of 3-D Structures for Microfluidic Devices", Sintering, (2005), pp. 3b6, 216-219.

10. Budniewski, K, et al., "Microchamber PCR device in LTCC - Modelling and Preliminary Experiments", Proc. VI Optoel. And Electronic Sensors Conf. (2004), pp.90-93.

11. Gongor-Rubio, M,R, et al., "LTCC manifold for heavy metal detection system in biomedical and environmental fluids", Proc. Conf. Eurosensors XVII (2003), pp.823-826.

12. Smetana, W, et al., "Processing Procedures for the Realization of fine Structured Channel Arrays and Bridging Elements by LTCC Technology", Microelectronics Reliability, Vol. 49 (2008), Issue 6, pp.592-599.

13. Deslandes, D, and $\mathrm{Wu}, \mathrm{K}$, "Single-Substrate Integration Technique of Planar Circuits and Waveguide Filters", IEEE Transactions on Microwave Theory and Techniques, Vol. 51 (2003), No. 2, pp. 593-596

14. Pan, B, et al., "Surface Micromachining PolymerCore-Conductor Approach for High-Performance Millimeter-Wave Air-Cavity Filters Integration", IEEE Transactions on Microwave Theory and Techniques, Vol. 56 (2008), No. 4, pp. 959-970. 